\title{
Body composition versus body fat percentage as predictors of posture/balance control mobility and stability among football players under 21 years
}

\author{
Zerf Mohammed
}

Physical Education Institute, University of Mostaganem, Mostaganem, Algeria

\begin{abstract}
Purpose:

Overhead athletes require a delicate balance of shoulder mobility and stability in order to meet the functional demands of their respective sport. While musculoskeletal symptoms (LBP) [1] is one of warning against the consequences recorded in abnormal posture, bending, twisting, gardening and exercise levels among the sportsmen. Based on this theorem, the current study aims to determine the strong factor which affects mobility and stability among soccer players under 21 years.

Material: 163 male players qualified from the Algerian football championship, engaged in the present study, selected by the intentional manner their average age 19,56 \pm 1.22 years. They were tested by saving tests (Body Fat Percentage (BFP) -Abdominal test (Abdo) - Modified Bass Test of Dynamic Balance (DB) and standing balance $(\mathrm{SB})$ ). As a statistical model, we chose regression analysis to predict the superiority of the variables chosen in the current study.

Results: $\quad$ Based on the analysis statistics, we confirm:

The high percentage of BFP is a great risk on the levels strengthens abdominal.

The maintenance of stability (SB) depends on the additional BFP associated with the BMI ratio as being higher factors predicting the total amount of body weight related to the risks of instability.

Maintaining dynamic mobility (DB) with respect to the displacement of the centre of gravity and its base support in the motion chain is based on excess body fat (BFP) in relation to body mass index (BMI) and Abdominal muscle strengthening levels (ABDO), as higher factors predicting the amount of weight gain.

Conclusions: Founded on the variances acquired by the search, we highlight the relationship between body mass index (BMI) and body fat percent (BFP) as betters index predicting the influence of total amount body weight on mobility and stability among soccer players up to 21 years age. Evidence guide us to recommend, the evaluation of body composition as a part of body management and control. Where strong relationships between BMI and BFP suggests the anomalies postural, muscle coordination, movement control, balance and awareness of body position in both the tests.

Keywords: Body composition, Body Fat Percentage, posture/balance control, players, football.
\end{abstract}

\section{Introduction}

Understanding control processes to maintain the stability or mobility of movements, it's an essential objective of studies that attempt to treat musculoskeletal system problems [2]. However, mobility has been reported in athletes as adaptive structural changes to the joint resulting in the extreme physiological demands of the activity. Whereas these structural adaptations compromise stability, exposing the athlete to shoulder injuries [3] set by clinical studies in LBP as higher prevalence among medical practitioners than any other musculoskeletal symptoms [4]. Thus, a sports studies indication the back pain as the frequent injury occurred in college football players (27\%), artistic gymnasts (50\%), and rhythmic gymnasts (86\%) [5]. While Laura Purcell, et al reported musculoskeletal symptoms [6] as a risk of the limited body alignment function related to the fix positions [7]. In case of the current study we referred to footballers, among whom low back injury was the most common and most common injury [8]. Based on the warning of health problems [9], which reports that the lifestyle experience decreased energy, after more than 25 minutes causing neck and back pain. Admit by Teen-Hang Meen, et al [10] in maintaining a standing posture for long

\footnotetext{
(c) Zerf Mohammed, 2017

doi:10.15561/20755279.2017.0208
}

periods, which leads to static muscular efforts, resulting in the back and headrest carriage, triggering to the musculoskeletal disorders [11], approved by prevalence studies in the significant musculoskeletal pain related to the quality of lifestyle [12]. The case of contact sports, repetitive flexion, extension, and torsion, which are the most injuries correlated to low back pain in the sports practises, suggesting athletic fitness as protective [13] of these injuries. Through this review of the literature, the present study was intended to investigate the impact of follow-up body mass index and body fat percent control as a predictor of these injuries. Moreover, in one hand, our hypotheses based on the confirmation, that the sports participation cannot guarantee physiological body mass and body composition [14], seen the BFP is not down to $5 \%$ among the athletes he/she wants to maintain or improve its performance [15]. Well, On the other hand, we criticise the procedure practised by the Algerian selectors which based on trainers' intuition, without taking into account anthropometric measurements [16]. Some similar studies avoid these methods and strategies and require the coach to use scientific methods [17]. The observed musculoskeletal symptoms require a specific program (evaluation and training).

On its foundations, our background theoretical based on medical studies, which admits that the human spine was 
not designed to be fixed [18], set by preventing studies in excess body fat to avoid the back problems. Furthermore, the current study aimed to examine the superiority of BFP Vs BMI factors to predict the effect of mechanical body composition on fixed and mobile balance among soccer players under 21 years. In order to provide baseline information for future analytical studies, our aims are to describe the correlation between body mass and fat control as part of the body-weight management and their consequence on posture/balance control in fixed and dynamic positions.

\section{Materials and methods}

Study Protocol and Subjects

Protocol

To achieve this goal, we based on the indication, which agrees that the prevalence of low back pain is associations with body fatness, fat distribution and height [19]. Where athletes are at greater risk of sustaining a lumbar (lower) spine injury due to levels of physical activity [20] injury as lots of stress undergoes to the spine, well the absorption of pressure, twisting, turning, and even the bodily impact among the football players. Therefore, sports participation cannot guarantee physiological body mass and body composition, and it is necessary to prescribe an exercise targeting body mass and fat control according to [14].

Subjects

The subjects were 163 male soccer players up to 21 years age in the Algerian football championship participated in the present study. Their average age was $19.56 \pm 1.22$ years. They were tested by saving tests (Body Fat Percentage-Abdominal Strength - Modified Bass Test of Dynamic Balance and standing balance). To exclude the effect of sex on data, all subjects are male. None of the subjects had historically of inscrutable visual defects, vertigo, motor paresis or sensory deficits. Participation in this study was voluntary to attend experience. Informed consent was obtained, and coaches signed a document.

\section{Testing Protocol}

Our choice is based on the indication that generally having poor posture and mechanics, the abnormal posture becomes apparent. Whereas examining posture in a static position allows an unobstructed view of all postures elements. Where the correct posture minimises stress on muscles, bones, and joints while incorrect posture places abnormal stress on these structures [21].

$\checkmark \quad$ Measurements of standing balance (SB)

- Objective

To monitor the development of the player's ability to maintain a state of equilibrium (balance) in a static position, see fig 1. It shows standing balance (SB).

- $\quad$ Required Resources

To undertake this test, you will require Stopwatch and an assistant.

To conduct the test:

o The player stands comfortably on both feet with their hands on their hips.

o The player lifts the right leg, places the sole of the right foot against the side of the left kneecap and closed both eyes.

o The assistant gives the command "GO", starts the stopwatch and the player raises the heel of the left foot to stand on their toes. The player is to hold this position for as long as possible.

o The assistant stops the stopwatch when the player's left heel touches the ground or the right foot moves away from the left knee. The assistance records the time.

$\checkmark \quad$ Modified Bass Test of Dynamic Balance (DB)

This multiple hop test requires that 1-inch $(2.5 \mathrm{~cm})$ tape squares are laid out in a course as shown in figure 1 . The subject is required to jump from square to square, in numbered sequence, using only one leg. The hands should remain on the hips. On landing, the subject remains to look face straight ahead, without moving the support leg, for five seconds before jumping to the next square. See fig. 2. Shows Dynamic Balance (DB).

o Scoring: the result is recorded as either a success or fail. A successful performance consists of hopping to each tape mark without touching the floor with the heel or any other part of the body and holding a static position on each tape mark for five seconds without exposing the tape mark.

$\checkmark$ Body Fat Percentage (BFP)

Body fat can be estimated from body mass index (BMI) in the current study, we used the formula for children: Adult Body Fat $\%=(1.20 \times$ BMI $)+(0.23 \times$ Age $)$ - (10.8 x gender $)-5.4$

Using gender male $=1$, female $=0$. [22] [23]. $\checkmark$ Abdominal tests (Abdo)

The abdominal test measures the muscular strength and endurance of the abdominal muscles and hip flexors for 1 min recording the number of repetitions practised by the athlete [24] [25].

\section{Statistical Analyses}

Data tests obtained shows, on one hand, that our soccer players are allocated according to them Body Fat Percentage Categories, Athletes and Fitness. As the body mass index our total sample is categories Underweight $=<18.5$, Normal weight $=18.5-24.9$ and Overweight $=25-29.9$. In other hand, all the variables chosen to study accepts the normal distribution and homogeneity. Presented as a means \pm standard deviation, Shapiro-Wilk and Levene test. See Table 1. All Regression analysis and the relationships analysed between independent variables and predictors are significant at $\mathrm{P} \leq 0.05$, confirmed by ANOVA one way, $\mathrm{T}$ and the positive Pearson autocorrelation expected by Durbin-Watson which is less than 2 and more than 1 . See Table 2,3 and 4.

\section{Results}

From the regression analyses relating Strength ABDO and the variables used in the current study Table 2, as Model 1, the program showed that BFP was able to explain the changes in the Strength ABDO, where F and T, R and Durbin-Watson are significant at $\mathrm{P}<0.001$. Through regression analysis relating standing balance (SB) and the 
variables used in the current study Table 3 model 1 and 2, the program showed that BFP and BMI were able to explain the changes in the Standing balance (SB), where $\mathrm{F}$ and $\mathrm{T} R$ and Durbin-Watson are significant at $\mathrm{P}<0.001$. While in regression analyses relating dynamic balance (DB) and the anthropometrics variables used in the current study Table 4. Model 1,2, and 4, the program showed that $\mathrm{BFP}, \mathrm{ABDO}, \mathrm{BMI}$ and weight were able to explain the changes in the dynamic balance (DB), where $F$ and $T R$ and Durbin-Watson are significant at $\mathrm{P}<0.001$. Although our results link with the Posture Committee of the American Academy of Orthopaedics in 1947, that posture is the regular and balanced arrangement of skeletal components to maintain the body's support structures against injuries and progressive deformation [26]. However, the function of the spine is divided between the anterior (static) and posterior (dynamic) [27], which mechanically required the intermediate of intrinsic skeletal muscles to protect the spinal column [28] the case of regression analyses Table 2. Whereas the minimal muscle strength in altered posture necessitates the dorsal muscles to improve more effort to maintain the balanced position [29], leading to fatigue, skeletal asymmetry and pain nociceptive stimuli related to extreme muscle strain to maintain posture, confirmed by the literature in the significant relationship between muscle shortness, waist and thigh circumferences, and postural balance type [30], the case of regression analyses Table 2, 3 and 4. Moreover, our results confirm that strong relationship between BMI and BFP are superiors to explain the change in abdominal Strength in stable or fixed positions, as well as mobile positions which required the abdominal Strength related to excess of weight, where these findings are in conformity with the American College of Sports Medicine as an appropriate intervention strategies for Weight Loss and Prevention of Weight Regain for Adults [31].

\section{Discussion}

The results of the present study based on the norms elaborate by the American Council on Exercise (ACE) as Ideal Body Fat Percentages [32] and BMI Categories according to the normative descript by the National Institutes of Health [33]. Whereas all the variables selected to study, shows that BFP and BMI are superiors to predict

Table 1. Shows descriptive Statistic baseline characteristics of the total sample.

\begin{tabular}{llllllllll}
\hline Variables & N & Mini & Maxi & Means. SD & \multicolumn{2}{l}{ VarianceShapiro-WilkP $\leq \mathbf{0 , 0 5}$} & Levene's & P $\leq \mathbf{0 , 0 5}$ \\
\hline Weight $(\mathrm{kg})$ & 163 & 53,56 & 79,00 & $65,05 \pm 6,15$ & 37,80 & 0,98 & 0,19 & 1,97 & 0,77 \\
Height $(\mathrm{cm})$ & 163 & 164,00 & 188,00 & $176,26 \pm 4,69$ & 21,98 & 0,99 & 0,28 & 0,11 & 0,66 \\
BFP $(\%)$ & 163 & 6,27 & 18,98 & $13,04 \pm 2,51$ & 6,28 & 0,99 & 0,58 & 0,20 & 0,18 \\
BMI (KG $\left./ \mathrm{m}^{2}\right)$ & 163 & 16,16 & 26,94 & $20,99 \pm 2,11$ & 4,42 & 0,97 & 0,19 & 0,10 & 0,33 \\
SB $(\min )$ & 163 & 2,02 & 8,81 & $4,04 \pm 1,13$ & 1,28 & 0,95 & 0,12 & 1,31 & 0,32 \\
DB $(\min )$ & 163 & 1,45 & 4,00 & $2,42 \pm 0,44$ & 0,19 & 0,96 & 0,18 & 1,24 & 0,18 \\
ABDO $\left(\mathrm{n}^{\circ}\right)$ & 163 & 25,00 & 56,00 & $36,48 \pm 6,34$ & 40,15 & 0,98 & 0,14 & 1,33 & 0,28 \\
\hline
\end{tabular}

Table 2: Presents the Results of regression analyses relating Strength ABDO and the anthropometrics variables used in the current study.

\begin{tabular}{|c|c|c|c|c|c|c|c|c|}
\hline $\begin{array}{l}\text { Model } \\
\text { STEPWISE }\end{array}$ & $\mathbf{R}$ & R 2 & Adjusted R 2 & Coefficients & $T$ & $\mathbf{P}$ & $\mathbf{F}$ & Durbin-Watson \\
\hline 1 & $0.23^{\circ}$ & 0.056 & 0.05 & $\begin{array}{l}\text { (Constant) } \\
\text { BFP }\end{array}$ & $\begin{array}{l}17,12 \\
-2,99\end{array}$ & $\begin{array}{l}0.00 \\
0.00\end{array}$ & 8,98 & $0.003^{b} 1,18$ \\
\hline
\end{tabular}

a. Dependent Variable: Strength ABDO

b. Predictors: (Constant), BFP

Excluded Variables: Weight, Height, BMI.

Table 3: Presents the Results of regression analyses relating standing balance (SB) and the anthropometrics variables used in the current study.

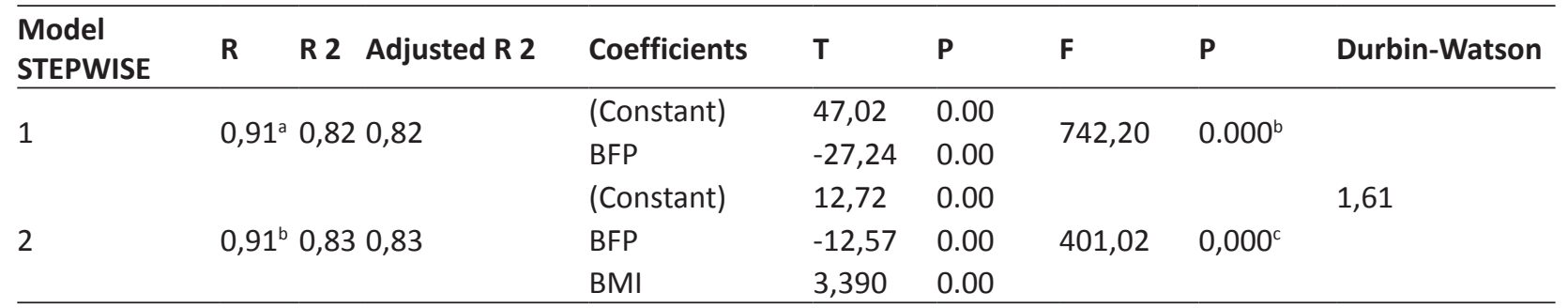

a. Dependent Variable: standing balance(SB)

b. Predictors: (Constant), BFP

c. Predictors: (Constant), BFP, BMI

Excluded Variables: Weight, Height and ABDO. 
Table 4. Presents the Results of regression analyses relating dynamic balance (DB)and the anthropometrics variables used in the current study.

\begin{tabular}{|c|c|c|c|c|c|c|c|}
\hline $\begin{array}{l}\text { Model } \\
\text { STEPWISE }\end{array}$ & $\mathbf{R}$ & R 2 Adjusted R 2 & Coefficients & $\mathbf{T}$ & $\mathbf{P}$ & $\mathbf{F}$ & Durbin-Watson \\
\hline \multirow{2}{*}{1} & \multirow{2}{*}{\multicolumn{2}{|c|}{$0,80^{\mathrm{a}} 0,650,64$}} & (Constant) & 39,26 & 0.00 & \multirow{3}{*}{295,21} & \multirow{3}{*}{$0.000^{b}$} \\
\hline & & & BFP & $-17,18$ & 0.00 & & \\
\hline \multirow{3}{*}{2} & \multirow{3}{*}{\multicolumn{2}{|c|}{$0,81^{\mathrm{b}} 0,660,65$}} & (Constant) & 15,70 & 0.00 & & \\
\hline & & & BFP & $-3,28$ & 0.00 & \multirow[t]{2}{*}{157,12} & \multirow[t]{2}{*}{$0,000^{c}$} \\
\hline & & & ABDO & $-2,71$ & 0.00 & & \\
\hline \multirow{4}{*}{3} & \multirow{4}{*}{\multicolumn{2}{|c|}{$0,82^{c} 0,670,66$}} & (Constant) & 15,06 & 0.00 & \multirow{4}{*}{109,59} & \multirow{4}{*}{$0,000^{d} 1,57$} \\
\hline & & & BFP & $-3,00$ & 0.00 & & \\
\hline & & & ABDO & $-2,71$ & 0.01 & & \\
\hline & & & $\mathrm{BMI}$ & $-2,360$ & 0.02 & & \\
\hline \multirow{5}{*}{4} & \multirow{5}{*}{$0,83^{d}$} & & (Constant) & 12,80 & 0.00 & \multirow{5}{*}{90,49} & \multirow{5}{*}{$0,000^{e}$} \\
\hline & & & BFP & $-2,95$ & 0.00 & & \\
\hline & & $0,690,68$ & ABDO & 3,39 & 0.00 & & \\
\hline & & & $\mathrm{BMI}$ & $-2,71$ & 0.00 & & \\
\hline & & & Weight & $-2,164$ & 0.03 & & \\
\hline
\end{tabular}

a. Dependent Variable: dynamic balance(DB)

b. Predictors: (Constant), BFP

c. Predictors: (Constant), BFP, BMI

d. Predictors: (Constant), BFP, BMI, Weight

Excluded Variables: Height.

reduced mobility and stability in football players under 21 years in the both balance \& strength abdominal. View these results, we approve that:

1. The sportsmen are not safe from the augmented of the abdominal fat area accumulation in the abdominal and pelvic regions as an amount of total body, confirmed in Strength $\mathrm{ABDO}$ as a decrease of the buttocks muscle strength, which negatively affects habitually body posture and habitually locomotion [34] the case exposed in all regression analyses Table 2, 3 and 4.

2. The overweight grass is associated with an increased body weight gain as a risk connected with poor balance at a higher risk of lower limb injuries [35]. In the case of the current study, we confirm:

A. Sports participation cannot guarantee the negative effects of body mass and body composition on the physiological roundness [14].

B. Physical fitness and health evaluated must base on various environmental factors, which need to be considered when we examine the effect of sports participation on body composition and fitness [36]. Mentioned by World Health Organization (WHO) in the use of existing settings such as the national situation and cultural habits for the prevention of overweight and obesity [37].

C. The stockpiling Fat mass in the middle parts of the hull affects the segmental velocity, degree of mobility, control of balance and decrease of posture stability [38] [39] in the both balanced tests.

D. Body pain accumulates in abnormalities posture, leads to the decrease of muscle coordination, control of movement, balance, and awareness of body position [40] confirmed in our case in reduce of abdominal Strength.

E. Soccer players with a maximum body fat and BMI are likely carrying excess abdominal fat in their abdomen associated with limitation of their level physical mobility and stability [41] [42].

F. The prevalence of overweight/obesity in soccer players request interventions of coaches and fitness trainers to monitor the weight changes related to fitness and health levels [43].

G. Well-evaluated and trained programs at high levels of intensity can achieve favourable changes in body composition during the careers of football players, which can confer benefits for performance and injury prevention [44].

According to the descriptions of health professionals and sports diet specialists, coaches needed to understand the dynamic energy balance and be prepared with effective and evidence-based food approaches to help athletes to achieve their body weight goals [45]. Where the previous confirmed the negative effect of overweight and fatness on physical fitness and the strong relationship between body mass index and body fat percentage suggests the further use of body mass index in adolescent soccer players [43]. Viewing these results, we recommend the control of weight changes which recurred from the coach to improve muscular strength and flexibility and decreasing body fat [46], as well as the need of exercise abdominal, back and pelvic muscles at least 30 minutes daily training programme sessions, without remembering the weight control programs. Moreover, this practice is absent in Algeria football clubs, where our players are 
nominated by the traditional method basing on the "gaze of coach" [47]. The inability of this method lies in Body Fat measurements, which cannot be predicted with the naked eye [16].

In conclusion, our results agree with the evidence that the practice of football requires follow-up on the progress of players through an evaluation program of body management, tracking the change of the weight respectfully to levels physical performance. Therefore, the amount of BFP related to BMI leads to poor posture associated with the fragility of the posture, as well as the weakness of musculoskeletal and balance as physical performance.

\section{Conclusions}

Our conclusions support our hypothesis, that body composition and body fat gain are superiors to predict the accumulated fat in the abdominal and pelvic regions at great risk of fatness confirmed by medical Algerian studies in the general population [48]. While the current study reports, that increase of body fat related to BMI ratios, accumulate around the midsection fat, influence the levels of Strength ABDO conducting to decrease of abdominal movement ability, posture control capacity, leading to weekly posture, fatigue, painful muscular tension, poor muscle tone and body joint's performance [49]. Whereas the level of BFP related to ratios BMI depends on the ability of body joints balance related to a combination of mobility and stability, which needed maximum endurance of trunk torque [50].

From the considering that the spine tasks lie in supporting the back muscles, ligaments, facet joints, body of the vertebrae and the disks [51], the current research recommended, the evaluation of body fat related to BMI in comparing to the optional physical fitness (mobility and stability), which allows coaches to assess and condition the elite performance and design evaluation and training programs [52]. Moreover, the dynamically balanced posture requires the gymnastic sessions to develop strength, balance, speed, suppleness, stamina and core body skills, as well as the effect of correcting muscle imbalances; improving posture, coordination, balance, strength, and flexibility correlated to the effectiveness muscle performance; dynamic trunk and extremity strength, coordination, and endurance.

However, these practices request from our coaches, in on hand to integrate exercise targeting body mass and fat control, more than 15-30 min to perform body alignment in the standing position as well as dynamic mobility related to establishing the centre of gravity in motion. Well, on the other hand, the necessity to evaluate the excess body composition, based on body fat percent as a control of weightiness related to optimal performance [53].

\section{Conflict of interests}

The author declares that there is no conflict of interests.

11. Suzanne C. Martin, Mary Kessler. Neurologic Interventions for Physical Therapy. Edinburgh: Elsevier Saunders; 2007.

12.Jeffrey I, Mechanick Robert, Kushner F. Lifestyle Medicine: A Manual for Clinical Practice. UK: Springer Shop; 2016.

13.Marianne S Gengenbach, Thomas E Hyde. Conservative Management of Sports Injuries. Sudbury, Toronto: Jones and Bartlett Publishers; 2007.

14.Pantelis Theodoros Nikolaidis. Elevated Body Mass Index and Body Fat Percentage Are Associated with Decreased Physical Fitness in Soccer Players Aged 12-14 Years, Asian J Sports Med, 2012;3(3):168-174,.

15.Sharon Plowman, Denise Smith. Exercise Physiology for Health, Fitness, and Performance. Philadelphia: Wolters Kluwer Health/Lippincott Williams \& Wilkins; 2008.

16.Zerf Mohammed, Houar Abelatif, Mime Mokhtar, Bengoua Ali. Traditional versus scientific method: the differences exist between selecting players, JPES, 2016;16(1):673 - 678,

17.Vijender Sharma. Principles and Methods of Teaching. UK: Sports Publications; 2011.

18.Christine Felstead. Yoga for runners. Champaign, IL: Human Kinetics; 2014.

19.Clifford Chao KS, Smith Apisarnthanarax, Gokhan Ozyigit. Practical essentials of intensity modulated radiation therapy. Philadelphia: Lippincott Williams; 2005.

20.University of Maryland Medical Center (UMMC), Low Back Pain in Athletes, Maryland. [Internet] 2016 [updated 2016 Jan 1; cited 2016 Dec 28]. Available from: http://umm. edu/programs/spine/health/guides/low-back-pain-in-athletes

21.Sandra J Shultz, Peggy A Houglum, David H Perrin. Excerpts the Correctly examine posture. [Internet] 2015 [updated 2015 Jan 1; cited 2016 Dec 28]. Available from: http://www. 
humankinetics.com/excerpts/excerpts/correctly-examineposture.

22.Janjic Jelena, Baltic ZM, Glisic Milica, Ivanovic Jelena, Boskovic Marija, Popovic Milka, Lovrenovic Mirjana. Relationship between Body Mass Index and Body Fat Percentage among Adolescents from Serbian Republic, Journal of Childhood Obesity, 2016;1(2):1-5.

23.Zerf Mohammed, Houar Abelatif, Mime Mokhtar and Bengoua Ali. Height versus Weight which Cassel Parameter Determine Pulmonary Functions Fitness among the Algerians Soccer Players, J Pulm Respir Med, 2016;6:353-359.

24.Ashok DrC. Test your physical fitness. Delhi: Kalpaz Publications; 2008.

25.Sanjay Parashar. Art of Abdominal Contouring: Advanced Liposuction. Place of publication not identified: Jaypee Brothers Medical P; 2015.

26.Fabris de Souza SA, Faintuch J, Valezi AC, Sant'Anna AF, Gama-Rodrigues JJ, de Batista Fonseca IC, de Melo RD. Postural changes in morbidly obese patients, Obes Surg, 2005;15(7):1013-6.

27.David G Borenstein, Sam W Wiesel, Scott D Boden. Low Back and Neck Pain: Comprehensive Diagnosis and Management. Philadelphia: Saunders; 2004.

28. Michael P Burke. Forensic Pathology of Fractures and Mechanisms of Injury: Postmortem CT Scanning. US: CRC Press; 2011.

29.Andersson GB, Ortengren R, Herberts ., Quantitative electromyographic studies of back muscle activity relatated to posture and loading, Orthop Clin North Am, 1977;8(1):8596.

30.Ragıba Zagyapan, Cihan Iyem, Ayla Kurkcuoglu, Can Pelin, Mustafa Agah Tekindal. The Relationship between Balance, Muscles, and Anthropomorphic Features in Young Adults, Anat Res Int, 2012;14:60-63.

31.I-Min Lee. Epidemiologic Methods in Physical Activity Studies. Oxford, New York: Oxford University Pres; 2009.

32.Lauren Jawno, Fran Schumer. Change4good : ten essentials for food, fitness and the good life. Bloomington: Authorhouse; 2012.

33.U.S. Department of Health \& Human Services. U.S. Department of Health \& Human Services. [Internet] 2016 [updated 2016 Jan 1; cited 2016 Dec 28]. Available from: ttps://www.nhlbi.nih.gov/health/educational/lose_wt/BMI/ bmicalc.htm

34.Luka Tunjic. Obesity, Gravity, Strength and Balance. US: Lulu Enterprises Inc; 2005.

35.Haff G Gregory, Triplett N Travis. Essentials of strength training and conditioning. Champaign, IL: Human Kinetics; 2016.

36.Clemens Drenowatz, Ronald P Steiner, Susanne Brandstetter, Jochen Klenk, Martin Wabitsch, Jürgen M. Steinacker. Organized Sports, Overweight, and Physical Fitness in Primary School Children in Germany, J Obes, 2013;9:352345.

37.Ruth SM Chan, Jean Woo. Prevention of Overweight and Obesity: How Effective is the Current Public Health
Approach, Int J Environ Res Public Health, 2010;7(3):765783.

38.Charles B Higgins, Albert de Roos. Ovid Technologies, Inc., MRI and CT of the cardiovascular system. Philadelphia: Lippincott Williams \& Wilkins; 2006.

39.Míriam Raquel Meira Mainenti, Érika de Carvalho Rodrigues, Juliana Flávia de Oliveira, Arthur de Sá Ferreira, Cristina Márcia Dias, and André Luís dos Santos Silva. Adiposity and postural balance control: Correlations between bioelectrical impedance and stabilometric signals in elderly Brazilian women, Clinics (Sao Paulo), 2011;66(9):1513-1518.

40.Sandy Fritz. Sports \& exercise massage: comprehensive care in athletics, fitness \& rehabilitation. St. Louis, Mo: Elsevier Mosby; 2013.

41.Ronald Ross Watson. Nutrition in the prevention and treatment of abdominal obesity. Burlington: Elsevier Science; 2014.

42.Paul Insel, Don Ross, Kimberley McMahon. Nutrition. Sudbury: Jones \& Bartlett Learning; 2016.

43.Nikolaidis PT. Physical fitness is inversely related with body mass index and body fat percentage in soccer players aged 16-18 years, Med Pregl, 2012;65(11-12):470-475.

44.Trexler, Eric T, Smith-Ryan Abbie E, Mann J Bryan, Ivey Pat A, Hirsch Katie R, Mock Meredith G. Longitudinal Body Composition Changes in NCAA Division I College Football Players, Journal of Strength \& Conditioning Research, 2017;31(1):1-8.

45.Melinda M. Manore. Weight Management for Athletes and Active Individuals: A Brief Review, Sports Med, 2015;45(1):83-92.

46.Jerrold S Greenberg, George B Dintiman, Barbee Myers Oakes. Physical Fitness and Wellness: Changing the Way You Look, Feel, and Perform. UK: Human Kinetics; 2004.

47.Zahner LE. The 12 elements of the success of the Swiss OlympicAssociation for the promotion of the success. Swiss; 2012.

48.George ABray, Claude Bouchard, Handbook of Obesity -Volume 1: Epidemiology, Etiology, and Physiopathology, Third Edition. US: CRC Press; 2014.

49.Mohammed Z, Ali B, Idris MM, Hamzaoui H, Messaliti L. Impact of fat mass distribution body shapes on muscles strength and the joints pain, BLDE Univ J Health Sci, 2016;1:81-88

50.Kim DH, Yoon WY. Effect of core program exercise for lumbar extensor strength and pain of the patient with chronic low back pain, Indian J Sci Technol, 2015;8(1):353-359.

51.John Ebnezar. Low Back Pain. New Delhi: Jaypee Brothers Medical Publishers (P) Ltd; 2012.

52.Cullen BD, Cregg CJ, Kelly DT, Hughes SM, Daly PG, Moyna NM. Fitness profiling of elite level adolescent Gaelic football players, J Strength Cond Res, 2013;27(8):2096-103.

53.Pantelis Theo Nikolaidis. Body mass index and body fat percentage are associated with decreased physical fitness in adolescent and adult female volleyball players, $J$ Res Med Sci, 2013;18(1):22-26. 


\section{Information about the author:}

Zerf Mohammed; http://orcid.org/0000-0001-5013-5446; mohamed.zerf@univ-mosta.dz; Physical Education Institute Laboratory OPAPS, University of Mostaganem; Mostaganem 27000, Algeria.

Cite this article as: Zerf Mohammed. Body composition versus body fat percentage as predictors of posture/balance control mobility and stability among football players under 21 years. Physical education of students, 2017;2:96-102. doi: $10.15561 / 20755279.2017 .0208$

The electronic version of this article is the complete one and can be found online at: http://www.sportedu.org.ua/index.php/PES/issue/archive

This is an Open Access article distributed under the terms of the Creative Commons Attribution License, which permits unrestricted use, distribution, and reproduction in any medium, provided the original work is properly cited (http://creativecommons.org/licenses/by/4.0/deed.en).

Received: 20.01.2017

Accepted: 30.01.2017; Published: 04.04.2017 